Valeria Merola

\title{
Il mito in scena: Endimione e Diana ne Gli amori della luna di Margherita Costa
}

\begin{abstract}
Nell'ultima opera di Margherita Costa, Gli amori della luna, è possibile individuare un'eco significativa della sua scrittura poetica precedente. È soprattutto il personaggio di Diana a ereditare i tratti delle figure femminili che la poetessa studia nei suoi canzonieri amorosi. Come le donne rappresentate nella Chitarra, la dea è un'amante di sdegno che investe l'innamorato della sua rabbia e della sua delusione per essere stata abbandonata. La cornice mitologica racchiude però una rappresentazione della vita di corte che Costa conosce molto bene. L'inserimento di elementi comici e spettacolari contribuisce a definire la teatralità della scrittura di Margherita Costa e collocarla opportunamente nella cultura barocca a cui appartiene.
\end{abstract}

The e-journal «altrelettere» is hosted at the URL: http://www.altrelettere.uzh.ch, in accordance with the Open Access Policy of the University of Zurich. Please cite this article as follows: V. MEROLA, Il mito in scena: Endimione e Diana ne Gli amori della luna di Margherita Costa, in «altrelettere», (2021), pp. 75-93, DOI: 10.5903/al_uzh-54.

(C) This article is licensed under a Creative Commons Attribution 2.5. Switzerland (CC BY-NC-ND 2.5). Please read the license terms on the website: http://creativecommons.org/licenses/by-nc-nd/2.5/ch/deed.en 
Il dramma Gli amori della luna è l'ultima opera pubblicata dalla poetessa romana Margherita Costa. Apparsa nel 1654 per l'editore veneziano Giuliani, la pièce è dedicata ai duchi di Brunswick-Lüneburg. ${ }^{1}$ Secondo la prassi editoriale dell'epoca, il testo è introdotto da alcune pagine dedicatorie in cui la poetessa incornicia l'opera all'interno di un contesto referenziale. Nell'indirizzare i suoi versi drammatici alle altezze serenissime di Giorgio Guglielmo, Ernesto Augusto e Giovanni Federico, Margherita esibisce il motivo topico della lamentatio sul proprio destino di esule insieme con quello della presunta modestia della sua produzione poetica. I due elementi sono costanti nella scrittura poetica di Costa: da una parte il tema della lontananza dalla città natale occupa uno spazio considerevole in ognuna delle sue opere, fino a una mise en abyme nella Selva di cipressi, in cui l'io lirico si manifesta nella sofferenza di Elisa infelice, che «ritorna al pianto, e fatta ombra cadente; / soffre del viver suo l'hore più estreme; / e 'n seno del martir, con tai parole, / del suo Destino si querela, e duole» (Costa 1640, 240). D’altra parte, quello della esibita modestia rispetto alla qualità dei propri versi è un fattore costante nelle opere di Costa, che lo desume da una tradizione che dalla lirica femminile cinque-seicentesca risale fino al topos classico della filatura come attività delle donne. Nelle opere di Margherita il binomio con le arti proverbialmente muliebri è però menzionato in chiave ironica, come non richiesta giustificazione della propria attività poetica. Dietro alla maschera del divertissement innocuo, la giocosa poetessa romana nasconde una poesia capace di toccare temi delicati, come il ruolo delle donne nella società, e non solo nel rapporto con gli uomini, ma anche riguardo al loro diritto ad affrancarsi dalla dimensione familiare e di dedicarsi alle arti (cfr. Costa Zalessow 2010).

Nelle pagine paratestuali del dramma i due atteggiamenti ricorrenti nella scrittura di Margherita - il lamento e la modestia - arrivano però a combinarsi, come se la lunga lontananza dalla città natale avesse contribuito alla sua scelta di «dispennare la penna» e avesse dato vita a un dramma di «rozzo stile», da «compatire con il sesso» (Costa 1654, 9), appunto. Seguendo quello che è un topos della poesia femminile di età moderna, ma che nelle sue pagine assume il valore di una marca di riconoscimento, dal valore anche ironico se non addirittura segno di una certa bizzarria, ${ }^{2}$ Costa abbina la sprezzatura - più $o$ meno sincera - nei confronti di un'opera frutto di una dichiarata inadeguatezza 
delle competenze e dello stile con il leitmotiv dell'inferiorità del sesso. Fingendo così di rinchiudersi nel perimetro di una poesia marginale e d'occasione, estranea ai codici del petrarchismo, ma anche non in linea con il contemporaneo marinismo, Costa rischia la taccia di stravaganza per assecondare la familiarità dei suoi versi con un complemento musicale e di rimanere fedele a un'identità al di fuori dell'ordinario. Pur accettando la classificazione che Jessica Goethals applica al corpus lirico di Costa, che vi legge «her self stylization as a "bizarre" writer» (Goethals 2017, 48) in relazione agli elementi comici della scrittura, non va sottovalutata la significativa componente stravagante di questa poesia, che si colloca volutamente ai margini del canone contemporaneo, rimarcando la propria posizione al limite e valorizzando la componente femminile. Sarebbe riduttivo considerare l'opera di Costa come inserita in «a burlesque world» (Goethals 2017, 52) e quindi leggerla solo in funzione di questo orizzonte comico e buffonesco, di cui sicuramente mostra di conoscere e adottare i codici, ma del quale certo non rimane prigioniera. La poliedrica scrittura della poetessa romana va invece interpretata alla luce di una definita identità letteraria, del suo compiacimento nel muoversi tra toni diversi, ma soprattutto della divertita - ma non per forza comica - consapevolezza della propria capacità di giocare su vari piani. L'esibizione di ignoranza è senz'altro riconducibile alla modalità retorica dell'excusatio non petita, con cui la poetessa, dando a vedere di volersi giustificare delle manchevolezze che potrebbero essere imputate ai suoi versi, cerca di assicurarsi un lasciapassare che le consenta di inserire la propria voce in un ambiente maschile, magari per tranquillizzare gli uomini rispetto al rischio di un'eventuale competizione, o solo per guadagnarsi un ascolto meno esigente.

Quello «sconcio e biasimevole Mostro, quale per esser spogliato d'ogni perfezzione, ad altro non devo rassembrarlo, ch'ad uno storpio e mal formato Nano», è il parto di un «rozzo ingegno» e la poetessa lo intitola La Chitarra per paragonarlo a uno strumento «vile», «quasi da tutti esercitato», che si andrà a confondere nel «concerto di variati istrumenti»3 (Costa 1638a). Proprio perché è ispirata dalla poesia, che nella sua visione è quasi una divinità, l'«Auttora» mantiene un atteggiamento umile, tale da portarla a disprezzare i suoi stessi versi: «non vi parranno dattili, o spondei, / ma scartacci da cuocer le frittate» 4 
(Costa 1638a). I suoi versi «schietti e malvestiti» (Costa 1638a, 4) non si curano dei giudizi dei critici: al contrario, Margherita Costa sembra vantarsi, con una mossa rivelatrice, del proprio disinteresse per i codici e l'etichetta e quindi di una scelta originale, con coerenza dicendosi pronta ad abbandonare la penna per dedicarsi alla sola arte del filare.

Se sulle rive dell'Arno e del Tevere la poetessa si era contraddistinta per aver «esercitato in vece dell'Ago, la Penna», ora, nell'introdurre l'ultima pièce, anziché «stabilirmi nell'Età quel nome, che nella gioventù tra gli applausi di sì grand'Alme acquistai» (Costa 1654, 6), rimpiange di aver smesso di scrivere.

Se è vero quanto dichiarato da lei stessa nell'appello ai lettori, Costa tornerebbe alle lettere dopo «havere abbandonato nello spatio di quattro anni la Penna», obbligandosi «sotto Cielo straniero» a «esercitar l'ingegno a diffesa di lingua nemica» (Costa 1654, 9): «ò fin qui sotto silentio anottata me stessa» (Costa 1654, 7). Grazie alla dedica ai duchi spera ora che la sua opera, portata alla luce «nel termine di quindeci giorni» (Costa 1654, 9), sfidando l’invidia da cui si sente circondata, possa rinascere nel segno di Diana. «Il trasporto di Diana dalle Selve a gl'Amori, darebbe campo ai maldicenti di detestare una Dea» (Costa 1654, 7-8), ma Margherita offre la sua opera ai nobili dedicatari per scongiurare «il vilipendio del mio Parto» (Costa 1654, 8). Al buio in cui la poesia di Costa è stata costretta risponde la luce lunare che suggella le parole di quello che è probabilmente il suo ultimo lavoro in versi: la poetessa chiede ai destinatari che accolgano sotto la loro protezione «gl'amori di Diana, e il Sogno d'Endimione» (Costa 1654, 8).

\section{L'emblema di Margherita}

Per quanto la figura mitologica della dea della caccia non sia particolarmente presente nell'opera della poliedrica autrice romana - dove compare solo a rappresentare il tormento di cui si sente preda la donna che sfugge al crudele «cacciatore» e rimane vittima delle armi «di Diana, e d'Amor» (Costa 1638a, 81) o nell'immagine lunare di Cinthia che «hebbe a' suoi dolci vezzi amico il Cielo» (Costa 1638b, 124) - in questo testo della maturità sembrerebbe diventarne quasi un emblema. Della «Selvaggia Dea», rappresentata mentre viene colpita dalle frecce di Amore, la poetessa condivide sicuramente la 
condizione di amante e in particolare di amante non ricambiata, con una raffigurazione che la include di diritto nella galleria di donne sdegnate dalla crudeltà dell'innamorato, tipica del suo repertorio amoroso (dalla Chitarra allo Stipo, passando per le Lettere amorose).

Il punto di contatto a mio avviso più interessante è nella combinazione della natura lunare di Diana con la sua nuova condizione di innamorata. Mentre insiste sulla contraddittorietà ossimorica dell'ardore che si impossessa del cuore gelido di Cinthia e della dea della caccia, che da inarrivabile oggetto del desiderio si trasforma in cacciatrice, la poetessa sovrappone la componente virile della sua ambigua identità alla meno evidente indole femminile. Le linee del maschile e del femminile corrono parallele nella scrittura poetica di Costa, che all'intreccio delle voci e delle identità dedica molto spazio in tutta la sua opera, arrivando, nelle Lettere amorose, a impersonare sistematicamente secondo il modello ovidiano delle Heroides - il doppio ruolo. 5

$\mathrm{Al}$ freddo della Ragione, che non a caso è una delle personificazioni presenti sulla scena, ${ }^{6}$ si aggiunge il calore del Senso, proprio come nella sua voluminosa produzione lirica precedente, che coniuga la messinscena di un personaggio femminile dominato dalla passione con la lucida e distaccata visione di chi gioca col proprio ruolo, guardando ad esso con ironia. Sulla scena degli Amori della luna le due divinità si affrontano contendendosi il trionfo sugli esseri umani, divisi tra costanza e fragilità, gloria e gioia.

Rag. Ove Ragion non è, tutto è follia.

Sens: Non amo Savij ne la reggia mia.

[...]

Rag: Cede il Senso à Ragione.

Sens: è vana l'opinione.

Rag: Il Senso è spirto frale.

Sens: Pur scote ogni mortale.

Rag: Non chi costante ha 'l petto.

Sens: Chi di gioie ha diletto.

Rag: Son le gioie del Senso un'ombra vana.

Sens: è Ragione al gioir sempre è lontana.

Rag: Horsù vedremo, qual tra noi più vaglia.

O il tuo Nume fallace, o de le glorie mie l'opra sagace.

Sens: In questo Monte sia, che tu Ragion confessi 
qual è la forza mia.

Rag: Qui, qui, fermiam il piede,

ch'ad onta del tuo ardir tosto vedrai, se più di me potrai.

Ove non è Ragion, l'opra è fallace.

Andar vogl'io a Diana, e l'insensato affetto detestar del tuo spirto, e del tuo petto.

Sens: Ed io col dardo mio sovra quest'Alme, e sovra questi Campi saettar vuò di gioia ogni desio (Costa 1654, II, 2, 45-46).

Mostrando le loro intenzioni nei confronti dei protagonisti e delle protagoniste, nella terza scena del secondo atto Senso e Ragione di fatto svelano le motivazioni all'origine della vicenda rappresentata. Come sottolinea l'Arietta posta in conclusione della scena, il Senso vuole che i petti «ardano» «di molli affetti» e che ogni cuore sia colto da «vaghi pensieri», affinché Amore possa trionfare sulla Ragione. La Ragione aveva espresso nella scena precedente la propria preoccupazione rispetto a un mondo dominato dall'amore e dall'ozio, lamentando come l'età presente fosse diventata preda del Senso e le anime vivessero «d'opinione», al punto che «puote un van pensiero / hoggi l'Impero haver d'ogni mortale» (COSTA 1654, II, 2, 46). Abbandonata la gloria e la fama, «chi tra pugne d'Amor rimane vinto» (ibidem, 47) è soffocato dall'ozio. Endimione, «d'ogni Pastor sublime», è caduto vittima delle mollezze del senso e «di sonno asperso ha il lume» (ibidem, 45). La Ragione vuole ora rivolgersi a Diana, perché abbandoni «l'insensato affetto», riportando tra gli uomini «l'opra sagace».

Volendo attribuire alla Diana protagonista degli Amori della luna la funzione di immagine emblematica di Margherita Costa si viene ad autorizzare anche una lettura della pièce come opera in qualche modo conclusiva e quindi riepilogativa della produzione della poetessa romana. È lei stessa a suggerire questa interpretazione quando, nella dedica del libro, ricorda di aver dato alle stampe quattordici volumi, cui ora segue questo «picciolo Parto del mio rozzo stile». ${ }^{7}$ Per quanto l'excusatio circa la poca raffinatezza dei suoi versi sia - come si è detto - una marca distintiva della scrittura di Costa, in quest'opera essa 
assume un valore peculiare, che la rende un unicum nella produzione dell'autrice.

Gli amori della luna sovrappone il dramma pastorale con la favola mitologica, riprendendo l'esperienza della Flora feconda, ma alle forme teatrali aggiunge la padronanza del genere lirico, della cui ampia esperienza porta traccia nella versificazione e nell'immaginario poetico di riferimento. L'opera risente dello sperimentalismo di Margherita, che nei suoi libri di versi spazia abilmente tra metri narrativi e misure leggere, dall'ottava al capitolo in terza rima, dall'idillio alla canzonetta chiabreriana, dal sonetto alla prosa. All'endecasillabo, cui nella favola pastorale è affidata l'azione scenica, si aggiungono versi brevi, come il settenario e il quinario. Il prologo recitato dall'Invidia adotta integralmente l'endecasillabo, per sottolineare la gravità del momento. Al contrario le ariette cantate da ninfe e pastori privilegiano il quinario, che meglio asseconda il ritmo del canto spensierato. D'altra parte, come ha suggerito Teresa Megale in occasione del convegno Margherita Costa, poetessa virtuosa, l'opera è molto probabilmente pensata per la musica. A provarlo sarebbe la struttura del libretto, che fa pensare a un melodramma, ma anche la coincidenza temporale con la messinscena e la pubblicazione dell'Ergirodo della sorella Francesca Anna (su testo di Giovanni Andrea Moniglia). La competizione tra le sorelle potrebbe pertanto essere all'origine della prova musicale di Margherita.

Come per la sperimentazione metrica, anche per il ventaglio tematico la poetessa attinge al repertorio allestito con la produzione precedente, che qui viene continuamente richiamata. Se, come ho cercato di dimostrare in uno studio di prossima pubblicazione, ${ }^{8}$ l'ispirazione poetica tende costantemente a una prospettiva teatrale, in quest'opera le varie linee tracciate nei libri delle rime trovano una possibile composizione. Nella definizione dei personaggi e delle dinamiche amorose che li legano Margherita sembrerebbe accogliere gli studi preparatori realizzati nei suoi canzonieri, dove gli amanti «di sdegno» o «di scherzo» paiono fornire infinite variazioni su un tema teatrale in via di costruzione (o definito sulla scena comica, che l'attrice conosce per esperienza personale). ${ }^{9} \mathrm{Al}$ laboratorio della poesia, in cui Costa prova le voci della sua ispirazione, segue qui lo spazio dell'opera teatrale, che consente a quei personaggi già sperimentati di trovare la propria definizione. 
In quest'ottica è particolarmente interessante osservare come proprio Diana sia protagonista di brevi apparizioni nell'opera poetica di Margherita, che la nomina nella Chitarra e nello Stipo, per poi intitolarle l'opera dedicata a Cristina di Francia, La selva di Diana. Nelle due raccolte fiorentine la dea compare in opposizione ad Amore, mentre nell'opera uscita a Parigi è protagonista di uno dei sonetti dedicati alla passione amorosa nei diversi giorni della settimana e rivolti Ad amante che parte dalla città mentre piove. Nel secondo sonetto, per il lunedì, la poetessa si rivolge a Cinthia, per dirle di aver smesso di seguire le sue orme. Ha addirittura iniziato a odiare il giorno a lei consacrato, perché il suo «bel Sole» «s'en giace / a me tolto fra l'ombre».

Cinthia, s'io fui de l'orme tue seguace hor quelle sdegno, ed al tuo Nume il giorno consacrato abborrisco, e prend'a scorno del dardo tuo, de l'arco tuo la face.

Oh dì per me funesto in cui s'en giace a me tolto fra l'ombre il lume adorno del mio bel Sole; ed in cui fa soggiorno tra l'ire a danno mio lo Dio di pace.

Principio, che m'annuntia opre non pie, augurio grave di mia sorte rea, apportator de le miserie mie.

Ah, che s'un tanto ardor l'anima ardea ben era d'uopo, inique stelle, e rie, che m'agghiacciasse la più fredda Dea (Costa 1647, 52).

Nel giorno della luna, però, l'amante presagisce un «augurio grave di mia sorte rea» e la sofferenza della passione le fa immaginare che sarebbe stato meglio essere agghiacciata dalla «più fredda Dea». Il binomio tra il fuoco della passione amorosa e il gelo lunare ispirato dal modello di Diana (a cui la donna della Chitarra prova a paragonarsi) trova perfetta corrispondenza nel mito messo in scena nell'ultimo testo pubblicato.

\section{Una cornice mitologica}


La favola mitologica trae la sua materia dalla diciottesima delle Heroides ovidiane, in cui Leandro racconta a Ero di come abbia ricordato alla luna dei suoi amori con Endimione, episodio a cui Ovidio rimanda anche nel terzo libro dell'Ars amandi. Margherita Costa potrebbe aver attinto direttamente anche alla versione di Apollonio Rodio, che iniziava ad avere successo nel Seicento. Il mito circolava negli ambienti artistici, come testimonia il celebre affresco di Annibale Carracci a Palazzo Farnese, ma era destinato a godere di maggiore fortuna a partire dalla fine del Seicento, quando sarebbe diventato soggetto di decorazioni pittoriche e opere musicali, come la serenata di Alessandro Scarlatti e poi quella di Pietro Metastasio. Come ha scritto Cristina Acucella, il mito di Endimione si lega però alla diffusione dei temi «orfici, neo-platonici e cabalistici» (ACUCELLA 2014, 1), ottenendo un notevole successo nell'ambito del petrarchismo quattro-cinquecentesco, soprattutto in relazione all' «esemplificazione del contatto tra l'anima e le sfere superiori dell'essere» (ACUCELla 2014, 3).

L'azione, divisa in tre atti, è introdotta da un prologo dell'Invidia, che entra in competizione con l'Amore e va a mischiarsi tra le dee, per impedire - «non sia che co 'l Pastor goda la Luna» - che Endimione e la dea possano godere «dolci i momenti, e dilettosi i giorni» (COSTA 1654, 11), ma invece far sì che siano sottoposti a «giochi del Fato, e scherni di Fortuna» (COSTA 1654, 12). L'Invidia promette di mostrare il proprio ardire contro gli dèi, confondendo il fuoco con il gelo e costringendo Diana a scendere dal cielo nella terra.

Tra le Dee mischierommi, e fia mio gioco mostrar contr' i Celesti anco l'ardire; e mio vanto saranno, e mio gioire pugnar co 'l gelo, e contrastar co 'l foco.

Diana, benché Dea, proverà duolo per lui, ch’in Latmo ha la magione eletto; e, se l'errare in Cielo è a lei diletto, sarà sua pena soggiornar nel suolo. (CosTA 1654, 12)

Mentre l'Invidia prepara lo spettatore all'imminente rovesciamento, perché «dove Gioia ha nido, Invidia ha sede» (CosTA 1654, 12), le condizioni per la creazione del gioco sono poste da Amore e Sonno, che nella prima scena del 
primo atto avviano una tenzone che li vedrà opporsi per tutto il dramma. Se anche «tra Mortali / scotendo l'ali / il tutto ponno / l'Amore e 'l Sonno» (COSTA 1654, 13), le due divinità agiscono con mezzi diversi: ai colpi di fuoco lanciati da Amore, il Sonno risponde con «armi di gelo», gli strali dell'uno trovano corrispondenza nel «liquor» dell'altro. Mentre la fredda Cinzia dovrà cadere vittima di Amore, l’innamorato Endimione cederà al sonno: la dea vedrà «cangiar lo strale, ond'ella il fianco adorna» (COSTA 1654, 14), ${ }^{10}$ mentre il curioso scrutatore dei moti lunari «le sue veggianti luci al sonno dia» (COSTA 1654, 15). ${ }^{11}$ Con il gusto per il capovolgimento e per la coincidenza degli opposti tipico della poesia barocca, Margherita Costa immagina una «Diana amante»e un Endimione preda dell'oblio, che mostrino la potenza di amore sul cuore e del sonno sull'anima: «l'un prende l'alma, e l'altro lega il core». Assecondando il piacere per le simmetrie e per i ribaltamenti, Amore e Sonno colpiscono i due protagonisti annullandone l'identità: mentre Diana viene colpita da una freccia di segno opposto a quelle scoccate da lei nelle battute di caccia, Endimione chiude gli occhi intenti a scrutare la luna e i suoi movimenti. Così come la dea della castità abbandona la ragione per cedere alla passione, il giovane pastore dimentica la propria curiosità per i moti lunari (non necessariamente indicativa di un interesse per la nascente nuova scienza) ${ }^{12}$ per cadere nell'oblio del sonno.

Sul rovesciamento insiste la presentazione dei personaggi, che appaiono, l'uno, ghiacciato e sprezzante della passione di cui ardeva e, l'altra, «preda di un mortale in terra». È Diana a ricordare all'amante come la situazione si sia capovolta e a rimproverarlo del cambiamento.

\footnotetext{
Ti sovvenga spietato quante volte mentisti, quante volte giurasti non più fiero a mie pene, non più crudo a miei mali ch'ardeva il petto tuo, e ne l'anima algente provavi ardor nocente, e divenuto amante eri di fido esempio alma costante. Ed hor fra tanto gelo neghittoso ti stai, e volgi a me sol per schermirmi i rai
} 
Allo stesso modo delle amanti di sdegno della Chitarra, Diana si comporta come un'amante abbandonata, che travolge con la sua rabbia e col suo furore l'uomo colpevole di non ricambiare il sentimento. Le donne protagoniste del primo canzoniere fiorentino maledicono gli amanti e lanciano invettive contro di loro, li invitano a godere del loro dolore - «godi dunque (spietato) al mio tormento, / godi alli strazij miei, à mie querele: / vanne, vanne (crudel) sarai contento / di vedermi morire à te fedele» (COSTA 1638a, 128) $)^{13}$ - o si lamentano degli inganni subiti: «spergiuro, a che ingannarmi, a che tradirmi / con tue lusinche, e con fallaci apprezzi? / perché di folle speme il Cor nutrirmi, / e piagarmi ad ogn'hor con mille vezzi?» (COSTA 1638a, 130). ${ }^{14}$

Forte della certezza che «mal s'accoppia / Cinthia al Dio de l'Amore» (COSTA 1654, I, 4, 23), Diana si aggirava tra i «Latmij sassi», quando si imbatté in un «huomo mortale, o boschereccio Dio» (COSTA 1654, 24). Gli stessi occhi che erano soliti contemplare la luna sono ora rivolti verso di lei come un doppio sole; ${ }^{15}$ la dea si stupisce nel riconoscere su di sé i segni della fenomenologia amorosa: «ohimè punger mi sento, / a un punto avampo, e gelo» (COSTA 1654, 24), meravigliandosi di aver abbandonato il cielo «per esser preda d'un mortale».

Dimmi crudo, che fai?

Su'l Ciel vagheggi de la Luna i Rai?

Ah che non più le Stelle preme di Cinthia il piede, ma tra mortali si raggira in terra (CosTA 1654, 24).

Alle richieste della dea il giovane pastore risponde specificando la natura del sentimento che lo lega a lei: «io sono Amante, / ma non d'Amor noioso» (COSTA 1654, 26). Endimione le dice di preferire il riposo alle fatiche d'amore e in questo modo sembra ereditare l'esperienza descritta nel canzoniere erotico, in cui gli amanti sono rappresentati nelle difficoltà delle passioni non corrisposte, delle separazioni, delle gelosie, dei tradimenti, dei sospetti, delle malattie d'amore. Mentre la dea ricorda con rimpianto la virtù che le aveva consentito di 
sfuggire alle fiamme, Endimione dà prova della saggezza che gli viene ispirata dal sonno, proponendo un ideale di amore che non allontani dalla vita e che non si confonda con la morte: «tu in preda de la morte ami di darti, / io di viver', e amarti. / Tu godi ne i tormenti, / ed io bramo i contenti, / ed ho per miglior voglia / del riposo il piacere, / che fra tanti martiri / con la noia d'altrui morir di doglia» (Costa 1654, 26-27).

L'oblio a cui lo conduce il sonno porta il protagonista a definire l'identità dell'amata: «s'ella è la Luna, a me non sembra il Sole» (Costa 1654, I, 5, 33). Affrancandosi dall'immaginario petrarchista e dall'identificazione codificata di Laura con il sole, Endimione propone un ideale contrario alle fatiche d'amore e alle sofferenze, in nome di una «commodità» raggiungibile attraverso la forza di volontà. Il pastore ha conquistato una condizione di equilibrio, uno «sciolto core» che gli consente di considerarsi un «agiato Amante», con «tepide voglie», per cui «io non peno, e pur'amo / at al par de l'Amore il Sonno bramo» (COSTA 1654, 21-22).

Tutto puote chi vuole:

e ben è vile il petto, che superar non osa

un van desio di fragile diletto

(CosTA 1654, 28).

Diversa la prospettiva di Diana, per cui «fatiche ha per diletto / un generoso petto» (CoSTA 1654, 30) e che in questo modo conferma di potersi inserire a buon diritto nella galleria di amanti sdegnate della Chitarra, per esempio quando conclude in questi termini un lungo duetto con l'innamorato che la invita ad accontentarsi: «resta dunque crudele, / che non son gli agi tuoi / altro che mie ruine,/ [...] a chi l'ombre desia, si neghi il Sole» (CosTA 1654, 31).

Alla disputa tra i due personaggi mitici fa da cassa di risonanza il gioco di schermaglie amorose delle ninfe e dei satiri che popolano la favola. Negli intermezzi tra le apparizioni di Diana ed Endimione la scena si riempie delle vicende boscherecce dei personaggi minori, che ampliano il ventaglio della rappresentazione delle dinamiche d'amore, sempre attingendo al repertorio comportamentale allestito nell'opera poetica. Alla decisione di Endimione di abbandonarsi al sonno fa seguito il monologo del Satiro, che sente «ne le vene 
/ di focoso desio mordaci pene» (COSTA 1654, I, 6, 36) e, dicendosi preda «d'un sbarbato Dio», tesse un elogio della sua barba. ${ }^{16}$

Se alle figure pastorali è affidata l'azione della pièce, tra gelosie e sofferenze amorose, a Diana ed Endimione, così come alle coppie Amore-Sogno e RagioneSenso, la drammaturga assegna dei veri e propri duetti, che si alternano ai monologhi delle singole voci. I duetti sono costruiti con lo scopo di mettere in scena la dialettica tra punti di vista diametralmente opposti e sono caratterizzati da rapidi scambi di battute, molto spesso organizzati su analoghe strutture sintattiche che mettono in evidenza la divergenza delle posizioni e che probabilmente svelano la destinazione musicale del testo poetico.

Il rapporto tra Endimione e Diana viene declinato fino alla separazione degli amanti, mentre il giovane continua a inseguire i piaceri dell'oblio e la dea prende consapevolezza di aver perso la propria identità - ora che ha deposto le sue armi e «scopre la piaga sua» (COSTA 1654, II, 1, 43) ${ }^{17}$ - rendendosi conto di non essere più venerata dalle ninfe. Diana si riconosce come una «Ninfa frale, / che cede ad un mortale», al cui sguardo è pronta a «disarmare il petto, e sottoporre il dardo» (CosTA 1654, III, 2, 68). L’incontro con l'amato, nella terza scena del terzo atto, trasferisce in una dimensione onirica l'azione che, in linea con quanto prospettato dal Sonno fin dall'inizio della pièce, può aspirare ad essere vissuta soltanto così. A Diana, che si presenta come «colei, ch'alterna de la notte i giri», il giovane pastore risponde con un invito ad addormentarsi e a preferire la quiete del sonno al tormento della passione, perché «chi non dorme al fin d'amor delira» (COSTA 1654, II, 3, 72).

È nell'Arietta tra la terza e la quarta scena del terzo atto che Endimione, prendendo lo strale di Diana, improvvisamente si sveglia dal suo sonno e dà avvio al racconto del sogno che lo ha allietato mentre dormiva. Al tentativo intrapreso dal pastore di interpretare l'apparizione del Sole sul suo carro, la Dea risponde rivelando «l'incognito mistero» in cui è racchiusa una profezia dal valore encomiastico. Endimione in sogno scopre il proprio legame con il sole, in modo che, nel finale dell'opera, le due entità divine potranno tornare a congiungersi e $\mathrm{i}$ «Bransvichi eroi» arriveranno ad estendere la loro fama «oltre i confini del riposto Mondo» (COSTA 1654, III, 4, 81), portando ovunque la virtù e la pace. 
In una rinnovata fiducia nella potenza d'amore, il sole e la luna finiranno con il ritrovarsi e la dea accetterà di deporre «de lo stral le noie» e di portare «di nobil dardo il cor piagato», perché «il Ciel così fe', sorte lo vole, / anco la Luna haver deve il Sole» (CosTA 1654, III, 5, 85).

\section{Il mondo comico della corte}

La vicenda del sole e della luna è però uno schema all'interno del quale Margherita inserisce altri elementi che contribuiscono a definire l’identità dell'opera in senso barocco. La presenza del comico è indubbiamente una cifra stilistica della drammaturgia di Costa, che nel suo teatro mantiene viva la memoria dell'esperienza con la commedia dell'arte e in particolare con le corrispondenti pratiche sceniche. Il comico incrina la lineare struttura del dramma mitologico e immette movimento in una favola che è costruita su una staticità di fondo e sarebbe altrimenti interrotta solo dai duetti dei protagonisti. Per quanto distribuito in vari momenti della vicenda e in situazioni legate alla dimensione boschereccia, il comico si concentra in alcune zone del testo, in cui si assiste a un vero e proprio affondo. La scena di matrice più apertamente comica è la quarta del secondo atto, esattamente nel cuore dell'opera, in cui «Ortichetta vecchia» rimpiange gli amori della sua gioventù. Mentre ricorda i tempi passati, la donna sottolinea la natura teatrale della sua presenza «anch'io calco le scene mascherata» - e invita il pubblico a ridere della sua storia. La funzione del personaggio non è quindi tanto il melanconico ricordo dei tempi andati, di cui però, «in capo a novant'anni e sette mesi», rievoca il «rubino» che «rise» sulle sue labbra, l'«oro» dei capelli e le «rose» delle gote (CosTA 1654, II, 4, 49-50). Il ruolo di Ortichetta è di portare in scena la pretesa inevitabilmente comica di continuare a vivere l'amore nonostante la vecchiaia, secondo un paradigma tipico della commedia dell'arte, a partire dalla maschera di Pantalone di cui sembrerebbe una versione femminile. Come tante donne anziane della Chitarra e secondo un topos della poesia barocca, anche Ortichetta si inserisce nel repertorio amoroso, arrivando a sfiorare l'osceno mentre chiede agli ascoltatori «vi è nessun che mi voglia? ardo d'amore» (COSTA 1654, II, 4, 52). 


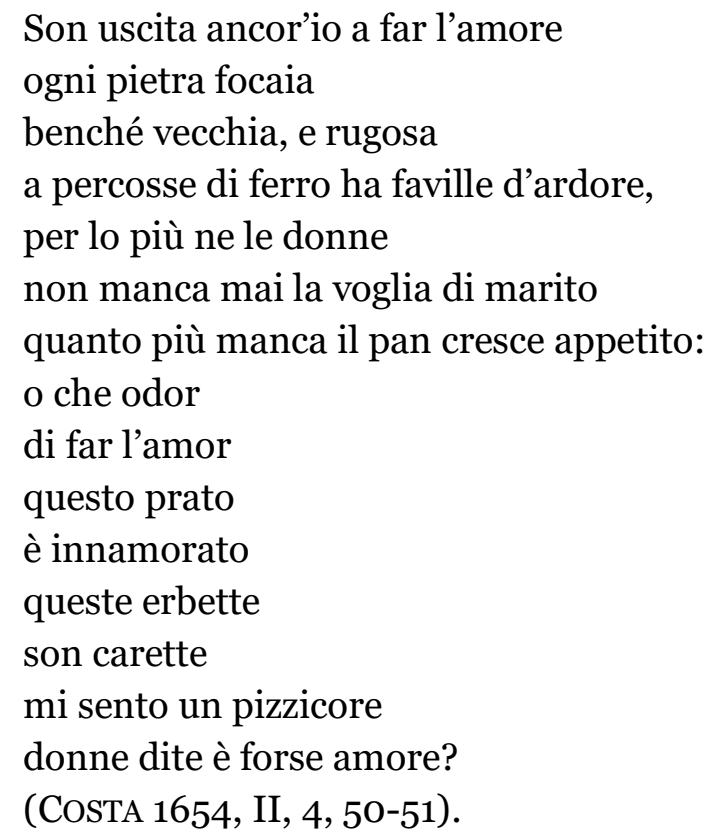

Se l'apostrofe alle donne come interlocutrici privilegiate rimanda ancora una volta alla Chitarra, dove pure si incontrano amanti non più giovani, l’invito a dedicarsi a un «canuto Amore» trova spazio soprattutto nelle Lettere amorose, dove Costa si muove con abilità tra toni comici e seri, con il distacco ironico necessario per prendersi gioco dei suoi stessi personaggi. È il caso della Risposta della Donna vecchia lunga sdentata all'Amante vecchio dentuto, in cui la caratterizzazione espressionistica stride con il tono apparentemente galante e leggero del madrigale che segue la prosa, e le parole di colei «ch'in vecchio ardore ha il sen canuto» appaiono comiche (COSTA 1639, 230). ${ }^{18}$

Un'altra significativa parentesi comica è offerta delle due scene di gioco che Margherita inserisce alla fine del primo e del secondo atto. ${ }^{19}$ Nello spazio pastorale, i personaggi si sottraggono alla tirannia di Amore organizzando giochi di società. «Chi non arde d'amor tra giochi posa» (COSTA 1654, I, 7, 38), osserva Dori, e intanto invita Ermilla e gli altri compagni a trovare pace con le carte nel Gioco della Staffetta. La scena, che sarà interrotta dall'arrivo del Satiro, rappresenta un momento di spensieratezza in qualche modo allusivo alla leggerezza della vita cortigiana di Margherita. Nel suo studio sul Gioco pratico, Raffaello Bisteghi parla in proposito del Gioco del cucù, che è «un Giuoco inventato a vero divertimento di numerosa Conversazione senza por in azzardo pericoloso i Giuocatori» (BISTEGHI 1760, 114). ${ }^{20}$ 
Alla fine del secondo atto ninfe e pastori si intrattengono invece nel Gioco degli Spropositi, in cui ognuno in silenzio dovrà pronunciare delle parole nell'orecchio del compagno, il quale a sua volta sarà invitato a «formar corrispondente unito senso» (CosTA 1654, II, 6, 61). Come scrive Agostino Mascardi, si tratta di un gioco diffuso «massimamente nella conversazione delle dame» (MASCARDI 1636, 618), che dimostra la necessità di un disegno logico nel favellare. Per Mascardi laddove questo manchi si assiste alla stessa dinamica del gioco, per cui la «scatenatura delle parti» determina la perdita della comunicazione e genera «indizi di follia» (BELLINI 2002, 220), come nella letteratura concettista. Se il tema del gioco offre un'ampia rappresentazione della vita della corte e della società della conversazione, ${ }^{21}$ nel caso degli Spropositi sembra esserci in effetti un'evidente allusione al concettismo barocco. Il "lieve ingegno" stimolato dal gioco deve portare alla creazione di mirabilia lessicali, ma anche di paradossi concepibili solo se dettati da amore. Come osserva Ermilla, si tratta di un gioco fatto «per ischerzo» (come gli amanti di scherzo della Chitarra, ancora una volta) che mette benevolmente in ridicolo la poesia d'amore, visto che «ove / amore ha il suo ricetto / non dar negli spropositi è difetto» (CosTA 1654, II, 6, 61).

Gioco, scherzo, paradosso e comico sono alcuni degli ingredienti degli Amori della luna, che si presta a porsi come lente attraverso cui leggere tutta l'opera di Margherita Costa. La pièce esibisce l'attenzione costante per il teatro che attraversa tutta la scrittura della poetessa romana, sempre rivolta a una dimensione spettacolare (la corte, la cornice, il repertorio comico). Ma è soprattutto attraverso questo specchio che possiamo collocare opportunamente la figura di Costa nella cultura barocca a cui appartiene. Se non bastasse a dimostrarlo l'applicazione del topos del mondo come teatro, più volte richiamato nel testo (e molto presente nella scrittura lirica dell'autrice), tale collocazione sarà invece evidente nella declinazione che di questa immagine viene proposta. È il Sonno a prevedere che «l'antro sia d'applausi a me teatro» (Costa 1654, I, 1, 15), prefigurando l'oblio di cui sarà vittima Endimione. Il Mondo di Margherita Costa «A me cede addormentato», in una combinazione di sogni e realtà che lo caratterizza inequivocabilmente come teatro barocco. 


\section{Note}

${ }^{1}$ Secondo quanto riportato da Dante Bianchi la poetessa avrebbe effettuato un breve viaggio in Germania, cfr. Bianchi 1924-1925, Capucci 1984 e Costa-Zalessow 2008.

${ }^{2}$ Cfr. Goethals 2017. Natalia Costa Zalessow parla di Margherita come della prima poetessa italiana a usare toni ironici e satirici, cfr. Costa-Zalessow 2015.

3 Le citazioni sono dalla dedica ai Duchi, senza indicazione di pagina.

${ }_{4}$ Margherita Costa, Capitolo scherzoso scusandosi l'Autora, in Costa 1638a (è la prima pagina numerata, ma la XXIV del volume).

5 A tal proposito Anna-Louise Wagner arriva a parlare di una scrittura ermafrodita per Margherita Costa (Wagner 2017). Lettere amorose si veda Piantoni 2017 e Piantoni 2018. Agli studi di Piantoni si deve soprattutto l'identificazione di una tendenza segnatamente teatrale dell'epistolario comico di Margherita Costa.

6 Sulle personificazioni di entità astratte e sul loro ruolo drammaturgico sulla scena moderna, rimando in particolare a Refini 2006.

7 Per quanto banale, si può notare come la metafora dell'opera letteraria come parto sia molto ricorrente nella scrittura paratestuale di Margherita Costa. Non sarebbe particolarmente rilevante se non fosse che sulla creatura poetica cade sistematicamente la sprezzatura dell'autrice. Nell'atteggiamento di esibito rifiuto dell'opera letteraria, la poetessa insiste sulla dimensione materna, probabilmente per enfatizzare l'innaturalezza del gesto, che così si rivela nella sua contraddittorietà. Non è da escludere poi che Costa giochi ancora una volta sugli stereotipi legati al femminile, combinando la maternità artistica con i ruoli muliebri sciorinati nei suoi versi.

${ }^{8} \mathrm{Il}$ saggio uscirà su «Esperienze letterarie».

${ }_{9}$ Cfr. Bianchi 1924-1925, Capucci 1984.

10 «A la Selvaggia Dea tra l'ombre io voglio / cangiar lo strale, ond'ella il fianco adorna; / e (di quella honestà vinto l'orgoglio) / amor destare, ove rigor soggiorna».

${ }_{11}$ «E co'l liquor, c'ho meco / io vuò sparger di Latmo il cavo speco; / e far, ch'Endimione, / che su l'alta maggione / i moti de la Luna intento spia, / le sue veggianti luci al sonno dia: / vanne tu dunque, e vanta / di far Diana amante. / Ch'io vuò col sonno mio / l'Amor d'Endimion sparger d'oblio; / e vedrem, chi più vale / o il valor del mio ramo, o del tuo strale».

${ }_{12}$ Già Boccaccio, nel capitolo De Luna, Yperionis filia della sua Genealogia deorum Gentilium afferma che Endimione «primus rationem cursus lune invenerit», cfr. Boccaccio 1998, VII, 408. Boccaccio sostiene anche che Endimione stesse in realtà meditando e che il suo meditare fosse scambiato per sonno.

${ }_{13}$ Bella Donna disdegnosa al suo Amante lungo tempo assente da Lei mentre appena tornato di novo ritorna a Partirsi, pp. 127-29.

14 Bella Donna si duole della crudeltà del suo Amante, pp. 130-32.

15 «Forse Apollo qui splende? / ma doppio è il Sol, che a gl'occhi suoi m'accende / Adon forse qui tace?».

16 Il satiro rappresenta un elemento di disturbo dell'equilibrio pastorale e bucolico del mondo delle ninfe e dei pastori, come è evidente per esempio in I, 8, quando Ermilla invita i compagni a uccidere o ferire il «novello mostro».

17 «Lascia il dardo, Diana, / deponi la faretra, e in vece sua / scopri la piaga tua. / Svela, svela ad altrui la tua ferita, / e dì, che Cinthia ancor serva è d'Amor, / e che la fredda Dea / ardendo chiede al suo gran Nume aita».

${ }_{18}$ Alla comicità del madrigale contribuisce anche il fatto che la poetessa costruisca i suoi versi sulla ripetizione sistematica degli aggettivi, per cui la risposta alla dichiarazione d'amore del «vecchio dentuto» avviene sull'imitazione: «Nessun dica, che giri / al mio canuto oblio / il tuo vecchio desio; / e se tu del mio crin l'argentee brine / brami in argenteo crine, / qual biasmo si può dare a vecchio ardore, / se per vecchia beltade arde d'Amore».

${ }_{19}$ Dante Bianchi considera i giochi «l'unica cosa che interessa, anche perché denota il diffondersi d'una costumanza», cfr. Bianchi 1924-1925, 207.

${ }^{20}$ Bisteghi 1760, Capitoli per il giuoco del cucù, 114-19.

${ }^{21}$ Alla vita di corte, che conosceva particolarmente bene dall'interno, Margherita Costa dedica diversi passaggi delle sue opere e in particolare la satira Cortegiano ravveduto, contenuta ne Lo stipo, sulla quale rimando alla pagina introduttiva di Costa Zalessow 2019. 


\section{Bibliografia}

\section{ACUCELLA 2014}

Cristina ACUCELlA, Luna, Endimione e la «morte nel bacio». Poetiche e filosofie a confronto in alcune declinazioni cinquecentesche del mito, "Griseldaonline», 14 (2014). Consultato il 30/09/2021, https://griseldaonline.unibo.it/article/view/9179.

\section{BELLINI 2002}

Eraldo BELLINI, Agostino Mascardi tra "ars poetica" e "ars historica”, Milano, Vita Pensiero, 2002.

BIANCHI 1924-1925:

Dante BIANCHI, Una cortigiana rimatrice del Seicento: Margherita Costa, in «Rassegna critica della letteratura italiana», 29, 1-31 (1924), pp. 187-203; 30 (1925), pp. 158-211.

\section{BISTEGHI 1760:}

Raffaello BISTEGHI, Il gioco pratico o sieno capitoli diversi che servono di regola ad una Raccolta di giuochi più praticati nelle Conversazioni d'Italia, Colle ameno, s.i.t.?, 1760 .

\section{BOCCACCIO 1998}

Giovanni Boccaccio, Tutte le opere, a c. di Vittore Branca, Mondadori 1998, vol. VII.

CAPPUCCI 1984:

Martino CAPPUCCI, Costa, Margherita, in Dizionario biografico degli italiani, Roma, Enciclopedia Treccani, 1984, vol. 30, pp. 232-34.

CosTA 1638a:

Margherita CosTA, La Chitarra, Francoforte, Daniel Watsch, 1638.

COSTA 1638b:

Margherita CostA, Il Violino, Francoforte, Daniel Watsch, 1638.

CosTA 1639a:

Margherita CoSTA, Lo Stipo, Venezia, s.i.t., 1639.

CosTa 1639b:

Margherita CosTA, Lettere amorose, Venezia, s.i.t., 1639.

CosTA 1640:

Margherita Costa, La Selva di Cipressi, Firenze, Massi\&Landi, 1640.

CosTA 1647:

Margherita CostA, La selva di Diana, Parigi, Craimoisy, 1647.

CosTA 1654:

Margherita Costa, Gli amori della luna, Venezia, Giuliani, 1654.

COSTA-ZALESSOW 2008:

Natalia Costa-ZALESSOW, Margherita Costa, in Albert N. MANCINI, Glenn Palen 
PIERCE, Dictionary of Literary Biography. Seventeenth-century italian poets and dramatist, Bruccoli, Clark Layman, 2008, vol. 339, pp. 113-18.

COSTA-ZALESSOW 2010

Natalia COSTA-ZALESSOW, Una poesia femminista del 1672 anonima e dimenticata, da attribuire a Margherita Costa, in «Esperienze letterarie», 35, 4 (2010), pp. 7985 .

COSTA-ZALESSOW 2019:

Natalia Costa-ZALESSOw, Cortegiano ravveduto: satira di Margherita Costa, «Letteratura italiana antica», XX (2019), pp. 579-91.

GOETHALS 2017:

Jessica GoETHALs, The Bizarre Muse: the literary Persona of Margherita Costa, in «Early modern women», 12, 1 (2017), pp. 48-72.

MASCARDI 1636:

Agostino MASCARDI, Dell'arte historica, Roma, Giacomo Facciotti, 1636.

PIANTONI 2017:

Luca PIANTONI, L'epistolario amoroso di Margherita Costa, in L'Italianistica oggi: ricerca e didattica, Atti del XIX Congresso dell'ADI - Associazione degli Italianisti (Roma, 9-12 settembre 2015), a cura di Beatrice Alfonzetti, Teresa Cancro, Valeria Di Iasio, Ester. Pietrobon, Roma, Adi editore, 2017, pp. 1-5.

PIANTONI 2018:

Luca PiANTONI, Le lettere amorose di Margherita Costa tra sperimentalismo e 'divertissement', in «Studi Secenteschi», 59 (2018), pp. 33-52.

REFINI 2006:

Eugenio REFINI, Prologhi figurati. Appunti sull'uso della prosopopea nel prologo teatrale del Cinquecento, «Italianistica», XXXV, 3 (2006), pp. 61-86.

WAGNER 2017:

Anna WAGNER, Amante monco a Donna senza naso": Margherita Costa's Baroque Parody of the Love Letter, Hull, Society for Italian Studies Conference, 2017 (conference paper). 